\title{
AN INVITATION TO THE STUDY OF UNIVALENT AND MULTIVALENT FUNCTIONS
}

\author{
A.W. GOODMAN \\ Department of Mathemat1cs \\ University of South Florida \\ Tampa, Florida 33620 \\ U.S.A.
}

ABSTRACT. We begin with the basic definition and some very simple examples from the theory of univalent functions. After a brief look at the literature, we survey the progress that has been made on certain problems in this field. The article ends with a few open questions.

AMS (MOS) SUBJECT CLASSIFICATION (1970) CODES. Primary 30A36, secondary 30A32, $30 A 34$.

1. THE HEART OF THE SUBJECT.

We are concerned with power series

$$
w=f(z)=\sum_{n=0}^{\infty} b_{n} z^{n}=b_{0}+b_{1} z+b_{2} z^{2}+\ldots
$$

in the complex variable $z=x+i y$ that are convergent in the unit disk 
$E:|z|<1$. Such a power series provides a mapping of $E$ onto some domain $D$. Two questions present themselves: (A) given the sequence of coefficients $b_{0}, b_{1}, b_{2}, \cdots$, what can we say about the geometric nature of $D:$ and (B) given some geometric property of $D$ what can we say about the sequence $\mathrm{b}_{0}, \mathrm{~b}_{1}, \mathrm{~b}_{2}, \cdots$ ?

An example of a nice geometric property is given in

DEFINITION 1. A function $f(z)$ that is regular (holomorphic) in $E$ is said to be univalent in $E$, if it assumes no value more than once in $E$. Such a function is also called simple or schlicht in E. When $f(z)$ is univalent in $E$ we say that the domain $D=f(E)$ is a simple (schlicht) domain.

Stated algebraically, $f(z)$ is univalent in $E$ if the equation $w_{0}=f(z)$ has at most one solution in $E$ for each complex $w_{0}$. If $f(z)$ is univalent in $E$, then $f^{\prime}(z) \neq 0$ in $E$. But one must be careful because the converse is false.

As trivial examples, we mention that $f(z) \equiv z$ is univalent in $E$ while $f_{2}(z) \equiv z^{2}$ is not univalent in $E$. The function $z+z^{n} / n$ is univalent in $\mathrm{E}$ for each positive integer $\mathrm{n}$. The function $\sin z$ is univalent in the larger disk $|z|<\pi / 2$.

PROBLEM. Find a useful set of conditions on the sequence $\left\{b_{n}\right\}$ that are both necessary and sufficient for $f(z)$ to be univalent in $E$.

This open problem is extremely difficult. However, partial results have been obtained, some of which will be stated here.

We observe that if $f(z)$ is univalent, then so is $f(z)-b_{0}$, and hence we may assume that $b_{0}=0$ in (1.1) without loss of generality. Geometrically this amounts to translating the domain $D$ so that $z=0$ goes into $w=0$ under the mapping $w=f(z)$. Next we note that $f^{\prime}(0)=b_{1} \neq 0$, so we can 
divide by $b_{1}$, and then write $f(z)$ in the form

$$
F(z)=z+\sum_{n=2}^{\infty} a_{n} z^{n}, \quad a_{n}=b_{n} / b_{1} \text {. }
$$

Geometrically this amounts to shrinking or expanding the domain D, and possibly rotating D. This does not disturb the univalence of the function.

When $F(z)$ has the form (1.2) we say that the function has been normalized. Other normalizations are possible, but the set of conditions $F(0)=0, F^{\prime}(0)=1$, is the most usual and the one we will use here.

We now give a very important example of a normalized univalent function. We start with the function

$$
g(z)=\frac{1+z}{1-z}
$$

and from the properties of the general linear fractional transformation, it is easy to see that $g(z)$ is univalent in $E$ and $g(E)$ is the half-plane $\operatorname{Re} g>0$. If we square $g(z)$ we see that the function $h(z) \equiv g^{2}(z)$ is also univalent in $E$ and maps $E$ onto the entire complex plane minus the slit along the negative real axis from 0 to $-\infty$. To normalize $E$ we subtract $h(0)=1$ and divide by $h^{\prime}(0)=4$. Thus we arrive at

$$
K(z) \equiv \frac{1}{4}\left[\left(\frac{1+z}{1-z}\right)^{2}-1\right]=\frac{z}{(1-z)^{2}}
$$

This important function is called the Koebe function and it maps $E$ onto the entire complex plane minus the slit along the negative real axis from $-1 / 4$ to $-\infty$. On intuitive grounds it is a "largest" univalent function because we cannot adjoin to $\mathrm{K}(\mathrm{E})$ any open set without destroying univalence. A short computation 
(starting with $\left.1 /(1-z)=\sum_{n=0}^{\infty} z^{n}\right)$ gives the power series for $K(z)$,

$$
K(z)=z+2 z^{2}+3 z^{3}+\ldots=\sum_{n=1}^{\infty} n z^{n}
$$

This power series for the "largest" univalent function suggests immediately CONJECTURE 1. If $F(z)$ is univalent in $E$, and has the power series (1.2), then

$$
\left|a_{n}\right| \leqq n
$$

for $n=2,3, \ldots$.

This conjecture has been the subject of research for more than 60 years and still represents an open problem, although it has been settled in many special cases. A complete account of these results is far beyond the scope of this paper but the interested reader can pursue the matter in the books by Spencer and Schaeffer [27], Goluzin [8], Jenkins [14], Hayman [11, 12], Pommerenke [25], and Schober [28].

The best result known today is due to D. Horowitz [13] who proved that

$$
\left|a_{n}\right| \leqq 1.0657 \mathrm{n}
$$

using a very deep method due to Carl FitzGerald [7].

The question raised by Conjecture 1, suggests a large number of related problems, some of which are open while others have been solved completely. The Koebe function is the "heart of the subject" because it appears so often, that a theorem in this subject becomes very interesting if it does not use the Koebe 
function or some modification of it. We will mention some of these theorems and conjectures, but first we give

\section{A SURVEY OF THE LITERATURE.}

The specialist in the theory of univalent and multivalent functions must be grateful to S. D. Bernardi, who has devoted much of his time to preparing an exhaustive bibliography of the subject [3]. The first volume covers the perfod from 1907 to 1965 and lists 1694 papers. The second volume covers the period from 1966 to 1975 and lists 1563 papers, Each of these 2 volumes has an extensive index which lists subtopics in this field and those papers that touch on each subtopic. Thus it is an easy matter for the specialist to determine the status of any problem up to the year 1975.

In addition, these two volumes give references to the many survey articles on the subject that have appeared during this period. Here we cite only three such; one by D. Campbell [5], a second by Anderson, Barth and Brannan [1, pp. 138-144], and third, the article by the author [9]. It is the purpose of this paper to review this third survey and bring it up to date by reporting on the progress made on the problems mentioned there.

3. THE PRESENT STATUS OF SOME OPEN PROBLEMS.

Let $S_{n}(z)$ be the $n^{\text {th }}$ partial sum of the series (1.2). Thus

$$
S_{n}(z)=z+\sum_{k=2}^{n} a_{k} z^{k}, \quad n \geqq 2 .
$$

QUESTION 1. Find necessary and sufficient conditions on the sequence $a_{2}, a_{3}, \cdots, a_{n}, \cdots$ such that $F(z)$ and every partial sum $s_{n}(z)$ is univalent in $E$. 
It is an easy matter to prove that if

$$
\sum_{k=2}^{\infty} k\left|a_{k}\right| \leqq 1
$$

then $F(z)$ is univalent in $E$. Indeed as A. Hurwitz first obseryed,

$$
\frac{F\left(z_{2}\right)-F\left(z_{1}\right)}{z_{2}-z_{1}}=1+\sum_{k=2}^{\infty} a_{k} P_{k}\left(z_{2} z_{1}\right)
$$

where $\left|\mathbb{P}_{k}\left(z_{2}, z_{1}\right)\right|=\left|z_{2}^{k-1}+z_{2}^{k-2} z_{1}+\cdots+z_{1}^{k-1}\right|<k$ for $z_{1}, z_{2} \varepsilon E$. It follows that if the condition (3.2) is satisfied then the right side of (3.3) is never zero for $z_{1}, z_{2} \varepsilon E$.

Now suppose (3.2) is satisfled, then the same inequality is true for each $S_{n}(z)$ and hence the condition (3.2) is a sufficient condition for $F(z)$ and every $S_{n}(z)$ to be univalent in $E$.

Let PS(1) denote the set of all functions $F(z)$ of the form (1.2) for which $F(z)$ and all the partial sums are univalent in $E$. Thus the condition (3.2) is a sufficient condition for $F(z)$ to be in PS(1). However, the condition is not necessary as we will soon see.

Alexander [2] has proved that if $a_{k} \geqq 0$ in (1.2) and

$$
1 \geqq 2 a_{2} \geqq 3 a_{3} \geqq \cdots \geqq n a_{n} \geqq \cdots \geqq 0,
$$

then $F(z)$ is univalent in E. For example

$$
-\ln (1-z)=\sum_{n=1}^{\infty} \frac{z^{n}}{n}
$$


satisfies the condition (3.4). In fact every partlal sum also satisfies (3.4) and hence - In $(1-z) \in \operatorname{PS}(1)$. Thus (3.4) is also a sufficient condition for $F(z)$ to be in PS(1). Further Alexander proved that if

$$
G(z)=z+a_{3} z^{3}+a_{5} z^{5}+\cdots+a_{2 k+1} z^{2 k+1}+\cdots
$$

and

$$
1 \geqq 3 a_{3} \geqq 5 a_{5} \geqq \cdots \geqq(2 k+1) a_{2 k+1} \geqq \cdots \geqq 0
$$

then $G(z)$ is univalent in E. Thus (3.7) is also a sufficient condition for a function $G(z)$ to be in PS(1). But neither of these conditions is included in the other, nor in the condition (3.2). The determination of all functions in PS(1) is a difficult task. Still more difficult is the generalization to p-valent functions.

DEFINITION 2. A function

$$
F(z)=\sum_{n=1}^{\infty} a_{n} z^{n}
$$

is said to be p-valent in a domain $E$ if it assumes no value more than $p$ times in $E$ and there is some $w_{0}$ such that $F(z)=w_{0}$ has exactly $P$ solutions in $E$, when roots are counted in accordance with their multiplicities. Observe that the normalization $a_{1}=1$ (used for univalent functions) has been dropped. Indeed it is no longer available because $f(z)=z^{2}$ is 2-valent in $\mathrm{E}$. 
Now let PS(p) be the set of all functions $F(z)$ such that $F(z)$ and all the partial sums $S_{n}(z)$, with $n>p$ have valence not exceeding $p$. The determination of PS(p) seems to be very difficult, and very little is known beyond the trivial $\mathrm{PS}(1) \subset \mathrm{PS}(2) \subset \mathrm{PS}(3) \cdots$. In this direction 0zak1 [24] has proved that if

$$
F(z)=z^{k}+\sum_{n=k+1}^{\infty} a_{n} z^{n}, \quad 1 \leqq k<p
$$

and if

$$
k(p-k+1) \geqq \sum_{n=k+1}^{\infty} n(n+p-1)\left|a_{n}\right|
$$

then $F(z)$ and all partial sums $s_{n}$, with $n \geqq k+1$ are at most p-valent in E. Thus $F(z) \in P S(p)$.

QUESTION 2. What can we say about $F(z)$, given by $(1.2)$ when $F(z)$ is biunivalent in $E$ ?

of course we need

DEFINITION 3. A function $F(z)$ is said to be biunivalent in $E$ if both $F(z)$ and its inverse are univalent in $E$.

Let us examine this definition a little more closely. On a first reading, it sounds as though $F(z)$ is 1-to-1, but 1-to-1 and regular is really univalent, so biunivalent must mean something different.

Suppose that $F(z)$ is regular in $E$ and $f^{\prime}(0)=1$. It follows from Schwarz's Lemma that either $F(z) \equiv z$ or there are two points $e^{i \alpha}$ and $e^{i \beta}$ such that $\left|F\left(e^{i \alpha}\right)\right|<1$ and $\left|F\left(e^{i \beta}\right)\right|>1$. The image of $E$ under such a 
function $F$ is shown in Figure 1. Let $\Gamma_{w}$ be an arc of the boundary of $F(\boldsymbol{F})$
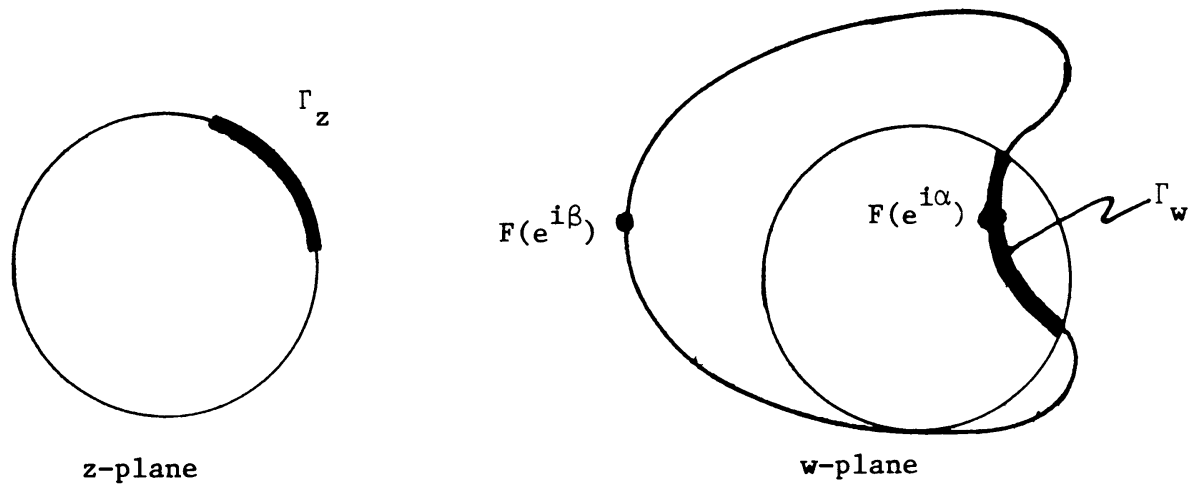

Figure 1

that lies inside $E$ in the w-plane and suppose that $\Gamma_{w}=F\left(\Gamma_{z}\right)$ where $\Gamma_{z} 18$ a suitable arc on $|z|=1$. If $F(z)$ is biunivalent, then it must be analytic on $\Gamma_{z}$ and such that it can be continued across $\Gamma_{z}$ so that $F^{-1}(w)$ is defined and regular throughout $|w|<1$. This additonal requirement is very stringent, and is very awkward to use in practice.

The concept of a biunivalent function was introduced by M. Lewin [19] who proved that if $F(z)$, given by (1.2), is biunivalent (belongs to the set BI), then $\left|a_{2}\right|<1.51$. At oṇe time, I believed that the bound $\left|a_{n}\right| \leq 1$ was true for every $n$, and that the extremal function should be $z /(1-z)=\sum_{n=1}^{\infty} z^{n}$. However, in a very difficult paper, E. Netanyahu [23] ruined this conjecture by proving that in the set $B I, \max \left|a_{2}\right|=4 / 3$.

Jenson and Waadeland [15] proved that if $f(z) \in B I$, then $\left|a_{3}\right| \leq 2.51$, but this result is not sharp (a lower bound may be the correct one).

Prof. Waadeland noted (in a letter to the author) that the interesting example on page 10 of [15] is not in the set BI because it has a pole at 
$w=16 / 25$, and that another example due to Busklein [4] has the same defect although it was intended to show that in $B I, \max \left|a_{n}\right|>n / 4$. At this time nothing is known about $\max \left|a_{n}\right|, \max \left|f^{\prime}(z)\right|, \max \left(\arg f^{\prime}(z)\right)$, etc, for the set of functions BI.

QUESTION 3. Let $A$ be the set of all functions of the form

$$
\phi(z)=\alpha f(z)+\beta g(z)
$$

where $\alpha, \beta \geq 0, \alpha+\beta=1$, and $f(z)$ and $g(z)$ are arbitrary functions from some given normalized set $X$. What can we say about $\phi(z)$ ?

First suppose that $X=S$, the set of normalized functions that are univalent in $E$. It is known [10] that if

$$
0.042 \approx \frac{1}{1+e^{\pi}}<\alpha, \beta<\frac{e}{1+e^{\pi}} \approx 0.958
$$

then it is possible to find $f$ and $g$ in $S$ such that $\phi(z)$ has infinite valence in $E$. However if $\alpha$, or $\beta$ are in the interval $\left(0,1 /\left(1+e^{\pi}\right)\right)$, then the maximum valence of $\phi(z)$ is unknown.

DEFINITION 4. We let $\mathrm{CV}$ be the set of all normalized univalent functions $F(z)$ for which $F(E)$ is a convex region. We let ST denote the set of all normalized univalent functions $F(z)$ for which $F(E)$ is starlike with respect to the origin. A domain $D$ is starlike with respect to the origin if the intersection of $D$ with each ray (starting at the origin) is either the ray itself or a line segment.

For example the functions $z$ and $z /(1-z)$ are convex (in CV), since $z /(1-z)$ maps $E$ onto the half-plane $\operatorname{Re} w>-1 / 2$. The Koebe function 
$z /(1-z)^{2}$ is not convex but it is starlike (in ST) because it maps $E$ onto the entire complex plane minus the slit $-\infty<w \leqq-1 / 4$. For the classes CV and ST, sharp coefficient bounds are known: If $f(z) \varepsilon$ CV then $\left|a_{n}\right| \leqq 1$ for every $n>1$ and $z /(1-z)=z+\sum_{n=2}^{\infty} z^{n}$ is an extremal function. If $f(z) \varepsilon S T$, then $\left|a_{n}\right| \leq n$ for every $n>1$ and the Koebe function is an extremal function.

It is clear from the definitions of $\mathrm{CV}$ and $\mathrm{ST}$ that

$$
\mathrm{CV} \subset \mathrm{sT} \subset \mathrm{s}
$$

We now return to question 3 .

Styer and Wright [29] have proved that if $\alpha=\beta=1 / 2$, then there are two functions $f$ and $g$ in ST for which $\phi(z)$ is infinite-valent. Such a conclusion may also hold for other values of $\alpha$ and $\beta$, but at present the range of such pairs in unknown.

In [9] Goodman conjectured that if $f$ and $g$ are in $C V$, then $(f+g) / 2$ is at most 2-valent. Styer and Wright [29] produced a pair of functions in CV for which $(f+g) / 2$ is 3 -valent and they venture the opinion that this sum "may very well be infinite-valent for some $f$ and 8 in $C V . "$ Other questions about such sums have been raised and answered. For further details see [5, 9].

There are similar results for the geometric mean of normalized functions,

$$
\psi(z)=f^{\alpha}(z) g^{\beta}(z) \equiv z\left(\frac{f(z)}{z}\right)^{\alpha}\left(\frac{g(z)}{z}\right)^{\beta},
$$

but for brevity we omit the details $[9,10]$.

We can replace the arithmetic or geometric mean in equations $(3,11)$ and (3.14) by other forms of composition. For this purpose we set 


$$
f(z)=z+\sum_{n=2}^{\infty} a_{n} z^{n}
$$

and

$$
g(z)=z+\sum_{n=2}^{\infty} b_{n} z^{n}
$$

and we define the two operators (convolution, Faltung) $f * g$ and $f * * g$ by

$$
H(z) \equiv f(g) * g(x) \equiv z+\sum_{n=2}^{\infty} a_{n} b_{n} z^{n},
$$

and

$$
J(z) \equiv f(z) * * g(z) \equiv z+\sum_{n=2}^{\infty} \frac{a_{n}^{b} n}{n} z^{n} .
$$

QUESTION 4. If $f$ and $g$ belong to the sets $X$ and $Y$ respectively, what can we say about $H(z)$ or $J(z)$ ?

As an example consider

CONJECTURE 2. If $f$ and $g \in S$ (normalized univalent function in $E$ ) then $f * * g$ is also in $S$.

If this conjecture were true, then it would be easy to prove (in several different ways) that $\left|a_{n}\right| \leqq n$ for every function in $s$. Unfortunately, conjecture 2 is false, and indeed has been disproved on several different occastons (see [6]). One easy approach is to observe that

$$
f(z) * * \frac{z}{1-z}=\sum_{n=1}^{\infty} \frac{a z^{n}}{n}=\int_{0}^{z} \frac{f(t)}{t} d t .
$$


Now Biernacki attempted to prove that if $f(z) \in S$, then the Integral in $(3,19)$ is also in $\mathrm{S}$. Unfortunately his proof was erroneous, and a counterexample was found by $\mathrm{Krzyz}$ and Lewandowski [17]. This same counterexample disproves the conjecture 2, but also disproves the much weaker conjecture: If $f \in S$ and $g \in C V$ then $f * * g$ is in $S$.

Suppose that $f$ and $g$ are in S. Since $J(z)$, given by (3.18) is not necessarily univalent, the question naturally arises, what can be said about the valence of $J(z)$ ? Campbell and Singh [6] gave the very surprising answer

THEOREM 1. There are two functions $f \in S$ and $G \in S T$ such that $f * * g$ has infinite valence in $\mathrm{E}$.

We outline the proof. By a theorem due to Libera [20], if $\phi(z) \varepsilon S T$ then

$$
g(z) \equiv \frac{2}{z} \int_{0}^{z} \phi(t) d t
$$

is again in ST. We apply this theorem with $\phi(z)=z /(1-z)^{2}$, the Koebe function, and find that

$$
g(z) \equiv \frac{2}{z} \int_{0}^{z} \sum_{n=1}^{\infty} n t^{n} d t=\sum_{n=1}^{\infty} \frac{2 n}{n+1} z^{n}
$$

is a starlike univalent function. Now take

$$
f(z) \equiv \frac{1}{1-b i}\left[(1-z)^{-1+b i}-1\right], \quad 0<b \leqq 1 .
$$

If we use the properties of the exponential function and the logarithm function, 
1t can be shown that $(1-z)^{-1+b i}=e^{(-1+b 1) \text { ln (1-z) }}$ is univalent in $E$, The other items in (3.22) merely normalize the function, Now

$$
J(z) \equiv f(z) * * \sum_{n=1}^{\infty} \frac{2 n}{n+1} z^{n}=\sum_{n=1}^{\infty} \frac{2}{n+1} a_{n} z^{n}=\frac{2}{z} \int_{0}^{z} f(t) d t .
$$

We apply this with $f(z)$ given by (3.22) and find that

$$
J(z)=\frac{2}{b i-1}\left[1+\frac{(1-z)^{b i}-1}{b i z}\right] \text {. }
$$

This function has infinite valence in $E$, because at the points $z_{n}=1-e^{-2 \pi n / b}, n=1,2, \cdots, J\left(z_{n}\right)$ always assumes the same value.

This theorem supplies one more counterexample for Confecture 2, and as far as the author can see, it is the simplest of the many counterexamples now known $[6,9]$.

Returning to question 4, we observe that different selections for $X$ and $Y$ and different properties for $H(z)$ and $J(z)$, yield a tremendous number of open problems. However the most interesting ones have been settled in a very important paper by Ruscheweyh and She1l-Small [26]. Here we merely state some of their results.

THEOREM 2. If $f(z)$ and $g(z)$ are normalized convex in $E$, then $H(z)$, given by (3.17), is also convex in $E$.

THEOREM 3. If $f(z)$ and $g(z)$ are normalized starlike in $E$, then $J(z)$ given by $(3,18)$ is also starlike in $E$.

THEOREM 4. If $f(z)$ is normalized starlike in $E$ and $g(z)$ is normalized convex in $E$, then $H(z)$, given by (3.17) is starlike in $E$. 
DEFINITION 5. Kaplan [16]. A function $f(z)$ of the form (3,15) is said to be close-to-convex in $\mathrm{E}$ if there is a univalent starlike function $\phi(z)$ such that in $E$

$$
\operatorname{Re} \frac{z f^{\prime}(z)}{\phi(z)}>0
$$

We let CC denote the set of all functions that are normalized and close-toconvex in $\mathrm{E}$.

Geometrically, the condition (3.25) implies that the image of each circle $|z|=r<1$ is a curve with the property that as $\theta$ increases the angle of the tangent vector does not decrease by more than $-\pi$ in any interval $\left[\theta_{1}, \theta_{2}\right]$. Thus the curve can not make a "hairpin bend" backward to intersect itself. Th1s means that each function in $\mathrm{CC}$ is univalent in E. Furthermore the class CC contains CV and ST. On the other hand there are univalent functions that are not in CC. Ruscheweyh and She11-Smal1 [26] extended Theorem 4 by proving:

THEOREM 5. If $f(z)$ is normalized close-to-convex in $E$ and $g(z)$ is normalized convex in $\mathrm{E}$, then $\mathrm{H}(2)$, given by (3.17) is close-to-convex in $E$.

THEOREM 6. If $f(z)$ is normalized close-to-convex in $E$ and $g(z)$ is normalized starlike in $E$, then $J(z)$ given by (3.18) is close-to-convex in $E$.

It is worthwhile to compare Theorem 1 and 6 and to observe that when "closeto-convex" is replaced by "univalent", the maximum valence of $f \star \star g$ jumps from 1 to $\infty$.

We now look at the bounds for the coefficients if $f(z)$ is $p$-valent in $E$. In his thesis, the author initiated 
CONJECTURE 3. If $f(z)=\sum_{n=1}^{\infty} a_{n} z^{n}$ is p-valent in $E$, then

$$
\left|a_{n}\right| \leqq \sum_{k=1}^{p} \frac{2 k(p+n) !}{(p+k) !(p-k) !(n-p-1) !\left(n^{2}-k^{2}\right)}\left|a_{k}\right|,
$$

for every $n>p$.

The reader will find in [9] some historical notes and an account of the progress on this conjecture up to the year 1968. When $p=1$, inequality (3.26) yields $\left|a_{n}\right| \leqq n\left|a_{1}\right|$ and this is equivalent to Conjecture 1. Certainly, we can hardly expect to prove (3.26) in full generality, as long as the inequality $\left|a_{n}\right| \leqq n\left|a_{1}\right|$ for univalent functions is still unsettled. However, there are some recent advances that are worth recording.

We generalized Definition 5 of close-to-convex univalent functions so that the new class $\mathrm{CC}(\mathrm{p})$ includes p-valent functions. Without going into too much detail, a function $\phi(z)$ belongs to the class ST(p) (starlike p-valent) if $f(z)$ is regular in $E$ and if there is some $r_{0}<1$ such that for each $r$ in $\left[r_{0}, 1\right)$ the 1mage of the circle $|z|=r$ under $f(z)$ is a curve that is starlike with respect to the origin $\left(\arg f\left(\mathrm{re}^{i \theta}\right)\right.$ is monotonic increasing in $D$ ) and the curve makes $p$ complete turns around the origin.

Now a function $f(z)$ is said to belong to the class CC(p) (close-toconvex of order p) if there is a function $\phi(z)$ in ST(p) and an $r_{1}<1$, such that

$$
\operatorname{Re} \frac{z f^{\prime}(z)}{\phi(z)}>0
$$

for $r_{1}<|z|<1$ (we omit some minor refinements here) [21].

The main point here is that $\operatorname{ST}(\mathrm{p}) \subset \mathrm{CC}(\mathrm{p})$ (any starlike function is 
close-to-convex) and hence any theorem about close-to-convex functions includes the same result about starlike functions as a special case. Two results by A. E. Livingston deserve mention.

THEOREM 7. [21]. Let $f(z)$ be close-to-convex of order $p$ in $E$. Then the inequality (3.26) is true for $n=p+1$, and this inequality is sharp In all of the varlables $\left|a_{1}\right|,\left|a_{2}\right|, \cdots,\left|a_{p}\right| \cdot$

In other words the bound for the coefficients of a p-valent function is true in the special case that $f(z) \varepsilon C C(p)$ and we look only at the (p+1)st coefficient.

THEOREM 8. [22]. Suppose that $f(z)$ is close-to-convex of order p In $E$, and that $a_{1}=a_{2}=\cdots=a_{p-2}=0$. Then the Inequality (3.26) Is true for every $n>p$ and every pair $a_{p-1}$ and $a_{p}$ not both zero. The Inequality is sharp in both varlables.

For the latest results about close-to-convex functions of order $p$, the reader should consult the paper by $R$. Leach [18].

\section{MORE OPEN PROBLEMS.}

Let A denote the set of all functions

$$
f(z)=z+\sum_{n=2}^{\infty} a_{n} z^{n}
$$

that are regular in $E$, and let $S$ denote the subset of those functions that are also univalent in $\mathbf{E}$.

It is not too difficult to ask questions about the set $S$, or questions about the relation of $S$ to $A$. Here some caution is necessary. When one considers the 3257 papers 1isted in Bernardi's bibliography together with those published since 1975, and those few that Bernardi may have missed, it is clear 
that a question selected at random may be answered already either explicitly or implicitly in some work previously published. With this note of warning to the reader we pose a few problems that we belleve are still open.

1. Suppose that $f(z)$ is in $S$ and that $f(E)$ is bounded by a rectifiable curve of length $L$. For fixed $L^{\star}>2 \pi$, let $S\left(L^{\star}\right)$ denote the subset of those functions for which $L \leq L^{\star}$. We can ask many questions about $S\left(L^{\star}\right)$. For example, in this set find $\sup |f(z)|$, find $\max \left|a_{n}\right|$ for each $n_{-}$If $f(z)$ omits $\gamma$ in $E$, find $\min |\gamma|$, etc.

2. Let $A$ denote the area of $f(E)$. For each fixed $A^{\star}$, let $S\left(A^{\star}\right)$ denote the subset of those functions in $S$ for which $A \leq A^{\star}$. We can ask the same type questions for this set that we asked about the set $S\left(L^{\star}\right)$. Here, we have the help of the well-known formula for the area of the Image of the disk $\mathrm{E}_{\mathbf{r}}:|\mathbf{z}| \leq \mathrm{r}$

$$
A=\pi\left(r^{2}+\sum_{n=2}^{\infty} n\left|a_{n}\right|^{2} r^{2 n}\right), \quad r<1,
$$

but there is no way to find an analogous formula for

$$
L=\int_{0}^{2 \pi}\left|f^{\prime}\left(r e^{i \theta}\right)\right| d \theta
$$

the length of the boundary of $f\left(E_{r}\right)$.

3. Let $\alpha$ and $\beta$ be fixed and suppose that $f(z)$ omits the two values $z_{1}=r_{1} e^{i \alpha}$ and $z_{2}=r_{2} e^{i \beta}$. What can we say about min $\left(r_{1}+r_{2}\right)$ for $f(z)$ in $S$ ? More generally if $\phi(s, t)$ is any fixed function what can we say about the minimum or maximum value of $\phi\left(r_{1}, r_{2}\right)$ for $f(z)$ in $s$ ? As a simple example, it is known that if $\beta=\alpha+\pi$, then 


$$
\frac{1}{r_{1}}+\frac{1}{r_{2}} \leq 4
$$

Suppose that $z_{1}$ and $z_{2}$ are fixed and $f(z)$ omits both $z_{1}$ and $z_{2}$ in $E$. If $f(z)$ is in $s$, find $\max \left|a_{n}\right|$ for each fixed $n>1$. Here, of course $\max \left|a_{n}\right|$ is a function of the omitted values $z_{1}$ and $z_{2}$.

4. As is customary, for $f(z)$ in A, set

$$
M(r, f)=\max _{\theta}\left|f\left(r e^{i \theta}\right)\right|
$$

for each $r$ in $(0,1)$ and let $M=\sup _{0<r<1} M(r, f)$.

If $f(z)$ is in $S$, it is well known that

$$
M(r, f) \leq \frac{r}{(1-r)^{2}}
$$

Suppose, conversely, we are given $M(r, f)$. Can we say anything about the valence of $f(z)$ ? What condition on $M(r, f)$ is sufficient to insure that $f(z)$ is univalent in $\mathrm{E}$ (or in some fixed smaller disk)?

5. If $f(z)$ is in $A$ and

$$
\operatorname{Re}\left(1+z \frac{f^{\prime \prime}(z)}{f^{\prime}(z)}\right)>0, \quad z \varepsilon E
$$

then $f(z)$ is univalent and $f(E)$ is a convex region.

If

$$
\operatorname{Re}\left(\frac{z f^{\prime}(z)}{f(z)}\right)>0, \quad z \in E
$$

then $f(z)$ is unfvalent and $f(E)$ is starlike with respect to the origin. 
Now in (4.7) and (4.8) replace 0 on the right side by - $C$ where $C$ is some fixed positive constant. We can no longer assert that $f(z)$ must be univalent. Can we give some upper bound on the valence of $f(z)$ as a function of the constant $\mathrm{C}$ ?

6. Let $M$ be some collection of functions each regular in $E$ (not necessarily univalent). The Koebe domain $D$ for the set $M$ is the largest domain $D$ that is contained in $f(E)$ for every function in the set $M$. For example, if $M=S$ then the Koebe domain is the disk $|w|<1 / 4$.

Koebe domains have been determined for a large number of different sets $M$, but we mention here two sets $M$ for which the Koebe domain is unknown. We first recall that a function $f(z)$ in $A$ is said to be typically-real if for all $z$ in $E$ we have

$$
(\operatorname{Im} z)(\operatorname{Im} f(z)) \geq 0
$$

This merely states that the upper half of the unit disk must go into the upper half-plane under $f(z)$, and the lower half of the unit disk must go into the lower half-plane. The interval $(-1,1)$ goes into the real axis. We let TR denote the set of all typically-real functions with the normalization (4.1).

The Koebe domain for the set TR is the domain bounded by the curve in polar coordinates

$$
\rho=\frac{\pi \sin \theta}{4 \theta(\pi-\theta)}, \quad 0<\theta<\pi
$$

together with its reflection in the real axis.

One open problem (suggested by E. Merkes) is to find the Koebe domain for the set of odd typically-real functions, with $f^{\prime}(0)=1$. 
Let $M>1$ be a fixed constant and let $T R(M)$ be the subset of functions in TR for which $|f(z)|<M$ for $z$ in $E$. Find the Koebe domain for the set $\operatorname{TR}(\mathrm{M})$.

7. Let $D_{1}$ be the domain common to the two disks $|z-i|<\sqrt{2}$ and $|z+i|<\sqrt{2}$. Goluzin first proved that every typically-real function is univalent in $D_{1}$ and that $D_{1}$ is the largest domain with this property. Thus $D_{1}$ is called the domain of univalence for the set TR.

For fixed $p$ find $D_{p}$ the domain of $p$-valence for the set $T R$. In other words find the largest domain $D$ such that every $f(z)$ in $T R$ has valence at most $p$ in $D$.

8. Finally we examine families of rational functions of the form

$$
f(z)=\sum_{k=1}^{n} \frac{A_{k}}{z-a_{k}}
$$

where the $A_{k}$ and $a_{k}$ are subject to certain restrictions.

As $P$. Todorov pointed out to me in a letter, Thale [30] was the first to prove that if $A_{k}>0$, and $-1 \leqq a_{k} \leqq 1$, then $f(z)$ is univalent in $|z|>1$ and this is a maximal domain of univalence for the family of functions that satisfy these conditions.

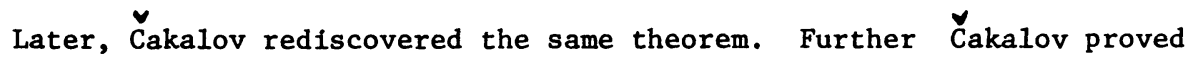
that if $A_{k}>0$ and $\left|a_{k}\right| \leq 1$, then $f(z)$ is univalent in $|z|>\sqrt{2}$, and this is the maximal domain of univalence.

Distler gave a beautiful generalization of these two theorems by finding the domain of univalence of the family (4.12) when the $a_{k}$ are restricted to lie in some fixed bounded set, and $\left|\arg A_{k}\right| \leq \theta_{0} / 2$ for some fixed $\theta_{0}<\pi$. 
If $D$ denotes the domain of univalence in any of the above families Todorov [31, 32] proved that one can add all of the boundary points of $D$ (with one exception) to $D$ without destroying the univalence of $f(z)$.

Suppose that in (4.11), we assume that

$$
-\infty<a_{1}<a_{2}<a_{3} \cdots<a_{n}<\infty
$$

and that all $A_{k}$ are real. Let $K_{j}$ be the closed disk

$$
\left|z-\frac{a_{j}+a_{j+1}}{2}\right| \leqq \frac{a_{j+1}-a_{j}}{2}, \quad j=1,2, \cdots, n-1 .
$$

Todorov [31] proved that, $f(z)$ given by 4.11 is univalent in $K_{j}-\left\{a_{j}\right\}$ for each $j=1,2, \cdots, n-1$.

As interesting as the above results may be, the really difficult problem is still untouched. Find $D_{p}$, the domain of $p$-valence, for each $p>1$ for the family of functions of the form (4.12) for some reasonable set of conditions on the constants $A_{k}$ and $a_{k}$. For this problem, I can not even suggest $a$ conjecture for $D_{p}$.

Finally we observe that Todorov [32] has investigated the applications of sums of the form (4.11) to magnetic fields. In this work, some of the residues $A_{k}$ may be negative.

\section{REFERENCES}

1. Anderson, J. M, K. F. Barth, and D. A. Brannan. Research Problems in Complex Analysis, Bul1. London Math. Soc. $\underline{9}(1977)$ 129-162.

2. Alexander, J. W. Functions which Map the Interior of the Unit Circle upon Simple Regions, Annals of Math. 17(1915) 12-22. 
3. Bernardi, S. D. A Bibliography of Schlicht Functions, New York University, Courant Institute of Mathematical Sciences, Part I 1966, Part II 1977.

4. Busklein. Spalteavbildinger og Schiffers randvariasjonsmetode, Hovedoppgave Norges Loererhogskole, 1968 .

5. Campbe11, D. A Survey of Properties of the Convex Combination of Univalent Functions, Rocky Mountain Jour. of Math. ㅁ(1975) 475-492.

6. Campbe11, D. and V. Singh. Valence Properties of the Solution of a Differential Equation, Pacific Jour. of Math., to appear.

7. FitzGerald, Car1. Quadratic Inequalities and Coefficient Estimates for Schlicht Functions, Archive for Rat. Mech. and Analysis 46(1972) 356-368.

8. Goluzin, G. M. Geometric Theory of Functions of a Complex Variable, Vol. 26, American Math. Soc. Translations, Providence, R. I., 1969.

9. Goodman, A. W. Open Problems on Univalent and Multivalent Functions, Bull. Amer. Math. Soc. 74(1968) 1035-1050.

10. - The Valence of Certain Means, Jour d'Analyse Math. 22(1969) $335-361$

11. Hayman, W. K. Multivalent Functions, Cambridge University Press, Cambridge, 1958.

12. - Research Problems in Function Theory, Athlone Press, London, 1967.

13. Horowitz, David. A Further Refinement for Coefficient Estimates of Univalent Functions, Proc. Amer. Math. Soc. 71(1978) 217-221.

14. Jenkins, James A. Univalent Functions and Conformal Mapping, SpringerVerlag, Berlin, 1965.

15. Jensen, E., and H. Waadeland. A Coefficient Inequality for Biunivalent Functions, Skrifter Norske Vid. Selskab (Trondheim), No. 15(1972) $1-11$.

16. Kaplan, W. Close-to-convex Schlicht Functions, Mich. Math. Jour. 1 1 (1952) 169-185.

17. Krzyz, J. and $\mathrm{Z}$. Lewandowski, on the Integral of a Univalent Function, Bull. de $1^{\prime}$ Acad. Pol. des Sci. 11(1963) 447-448.

18. Leach, Ronald. On the Goodman Conjecture for Close-to-convex Functions, Pacific Jour. of Math., to appear.

19. Lewin, M. On a Coefficient Problem for Biunivalent Functions, Proc. Amer. Math. Soc., 18(1967) 63-68. 
20. Libera, Richard, Some Classes of Regular Univalent Functions, Proc. Amer. Math. Soc., 16(1965) 755-758.

21. Livingston, A. E. p-valent Close-to-convex Functions, Trans. Amer. Math. Soc., $115(1965)$ 161-179.

22. - The Coefficients of Multivalent Close-to-convex Functions, Proc. Amer. Math. Soc. 21(1969) 545-552.

23. Netanyahu, E. The Minimal Distance of the Image Boundary from the Origin and the Second Coefficient of a Univalent Function in $|z|<1$, Archive for Rat. Mech. and Analysis 32(1969) 100-112.

24. Ozaki, S. On the Multivalency of Functions, Science Reports of the Tokyo Bunrika Daigaku, Section A, Vo1. 2, No. 36(1934).

25. Pomnerenke, C. Univalent Functions, Vanderhoeck and Ruprecht, Gottingen, 1975.

26. Ruscheweyh, S. and T. Sheil-Small. Hadamard Products of Schlicht Functions and the Polya-Shoenberg Conjecture, Comm. Math. Helvetici 48(1973) 119-135.

27. Schaeffer, A. C. and D. C. Spencer. Coefficient Regions for Schlicht Functions, Amer. Math. Soc. Coll. Pub. $\underline{35}$, Providence, Rhode Island, 1950.

28. Schober, Glenn. Univalent Functions - Selected Topics, Lecture Notes in Mathematics 478, Springer Verlag, Berlin, 1975 .

29. Styer, D., and D. Wright. On the Valence of the Sum of Two Convex Functions, Proc. Amer. Math. Soc. 37(1973) 511-516.

30. Thale, J. S. Univalence of Continued Fractions and Stieltjes Transforms, Proc. Amer. Math. Soc. 7 (1956) 232-244.

31. Todorov, P. On the Theory of Univalent Conformal Mappings that are Realizable by Meromorphic Functions with Simple Poles and Positive Residues (Russian), Ukrainian Math. Jour. 22(1970) 416-422.

32 . - Maximal Domains of Univalence of Certain Classes of Meromorphic Functions, Aequationes Math. 10(1974) 177-188.

33. Wassilev, T. and P. Todorov, Zur Mathematischen Theorie des Magnetischen Feldes des Systems Linearer Gleichstrome, Rev. Roumaine de Phy. $\underline{16}(1971)$ 543-546. 


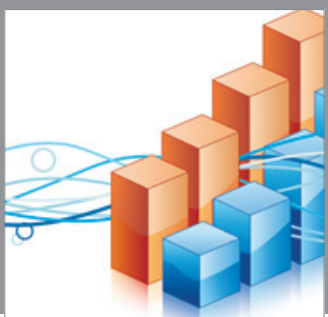

Advances in

Operations Research

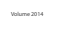

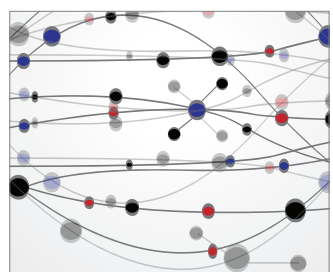

\section{The Scientific} World Journal
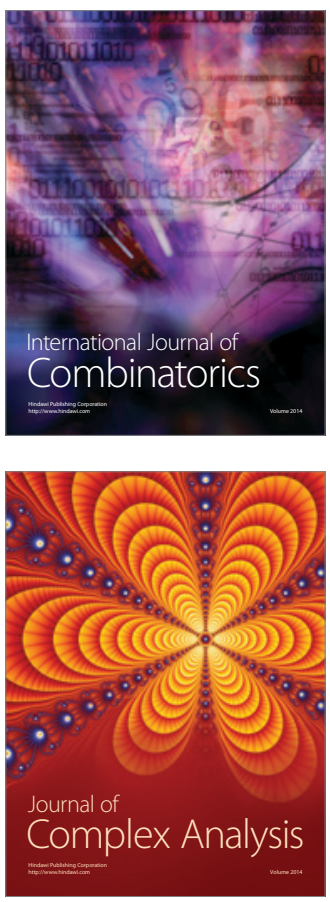

International Journal of

Mathematics and

Mathematical

Sciences
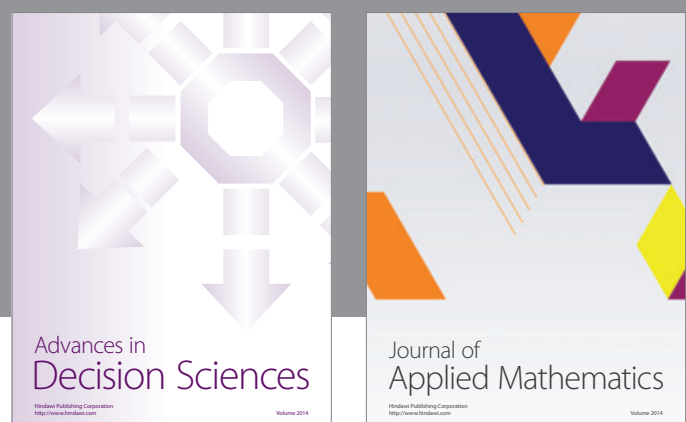

Journal of

Applied Mathematics
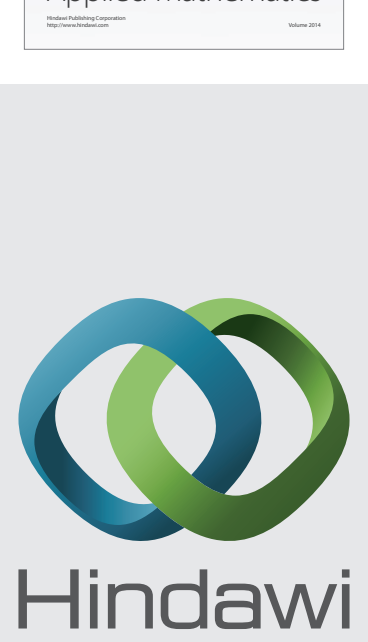

Submit your manuscripts at http://www.hindawi.com
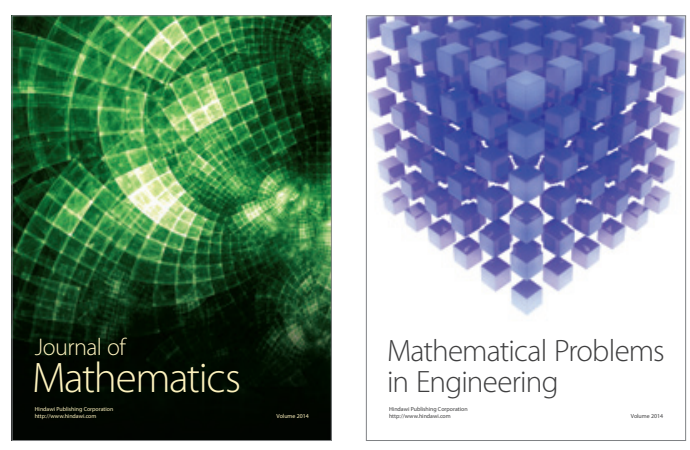

Mathematical Problems in Engineering
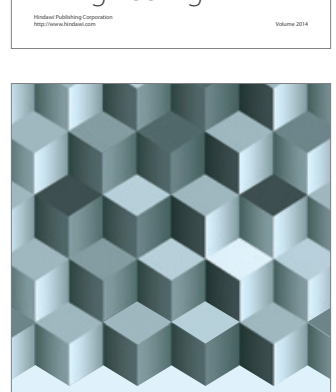

Journal of

Function Spaces
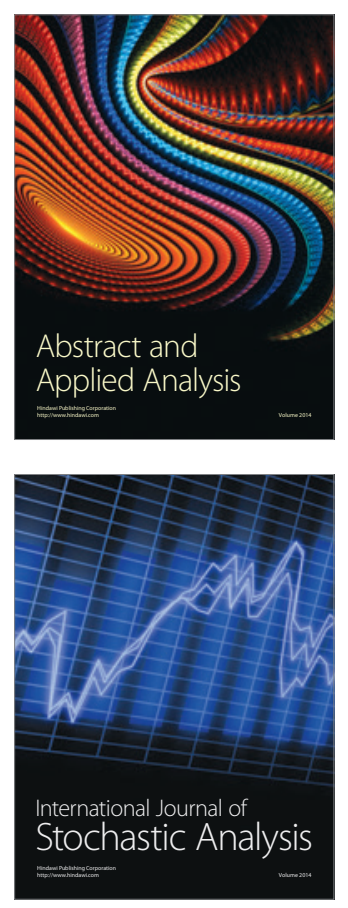

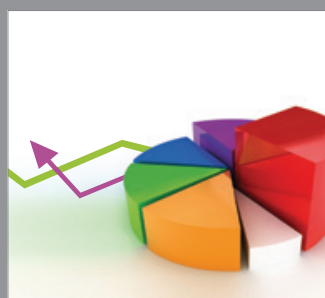

ournal of

Probability and Statistics

Promensencen
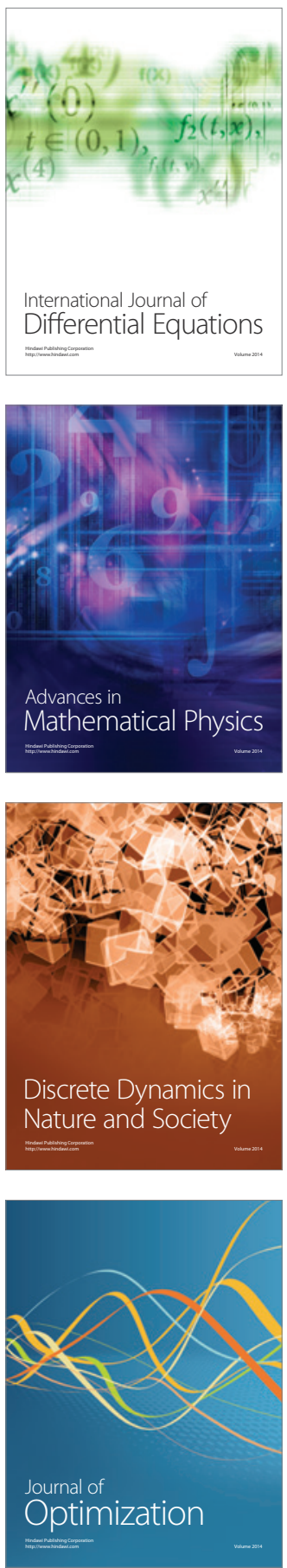